\title{
Gambian Trypanosomiasis
}

National Cancer Institute

\section{Source}

National Cancer Institute. Gambian Trypanosomiasis. NCI Thesaurus. Code C35084.

Trypanosomiasis caused by infection by Trypanosoma brucei gambiense. 